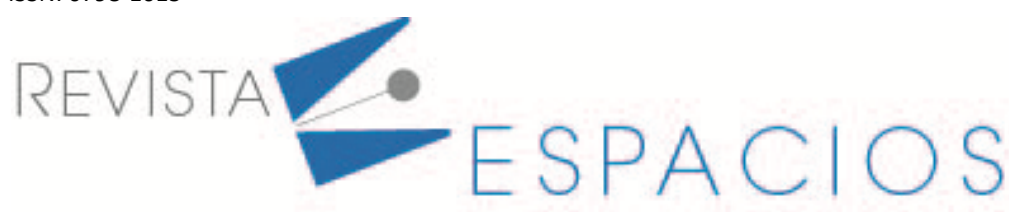

\title{
Valoración del apoyo al desarrollo del agroturismo sostenible a través del apego a la comunidad, las percepciones y la participación de los residentes
}

\section{Assessment of support for the development of sustainable agrotourism through community attachment, perceptions and participation of residents}

TAVERAS, José M. ${ }^{1}$

MORAL-CUADRA, S. ${ }^{2}$

\begin{abstract}
Resumen
República Dominicana es un destino consolidado en turismo de sol y playa, aunque los ingresos por ese concepto no han aliviado la pobreza. La industria agrícola es una de las más importantes para el país. Se presenta un estudio cuantitativo dirigido a los residentes de la región noroeste. El objetivo principal de esta investigación es proponer un modelo basado en ecuaciones estructurales donde se analizará si el apego a la comunidad, las percepciones agroturísticas positivas y la participación en la conservación tienen relación con el apoyo al desarrollo del agroturismo sostenible.

Palabras clave: Agroturismo, apego a la comunidad, participación, percepción
\end{abstract}

\begin{abstract}
The Dominican Republic is a consolidated destination for sun and beach tourism, although tourism revenues have not alleviated poverty. The agricultural industry is one of the most important for the country. A quantitative study aimed at residents of the northwest region is presented. The main objective of this research is to propose a model based on structural equations where it will be analyzed if the attachment to the community, the positive agro-tourist perceptions and the participation in the conservation are related to the support to the development of sustainable agro-tourism.
\end{abstract}

Key words: Agrotourism, community attachment, participation, perception

\section{Introducción}

El agroturismo es una modalidad turística que incluye una relación estrecha entre el espacio, el mundo agrícola y el turismo (Paül Carril y Araújo Vila, 2012). Estas características hacen que el agroturismo sea una modalidad propia, diferente a otras como el turismo rural (Ivars, 2000). Se ha comprobado que el agroturismo tiene beneficios potenciales para las comunidades (Zumbado-Morales, 2010). Actualmente, y en algunos destinos, las comunidades locales han desarrollado el agroturismo como fuente de ingresos (Little y Blau, 2019).

\footnotetext{
1 Profesor de la Universidad Tecnológica de Santiago, UTESA, Recinto Mao. Departamento de Turismo. Universidad Tecnológica de Santiago, República Dominicana. Correo electrónico de contacto: josemanueltaveras@gmail.com

2 Profesor de la Universidad de Córdoba. Departamento de Estadística, Econometría, Investigación Operativa, Organización de Empresas y Economía Aplicada. Universidad de Córdoba, España. Correo electrónico de contacto: salvador.moral@uco.es
} 
El agroturismo sostenible tiene un triple objetivo (Zhen et al., 2005): garantizar la autosuficiencia, conservar los recursos naturales y, además, desarrollar la prosperidad económica del destino. Así, el agroturismo permite a las comunidades locales generar ingresos a través de la agricultura para preservar los estilos de vida rurales, al tiempo que proporciona nuevas oportunidades para las generaciones más jóvenes (Little y Blau, 2019).

En relación con los aspectos económicos, el agroturismo fomenta la creación de empleo y el alojamiento, además de mayores ganancias al vender productos locales directamente a los turistas (Barbieri, 2013). En términos sociales, incrementa el estatus social de los agricultores, mejora la posición social de las mujeres y fomenta la conservación de las costumbres locales (McGehee y Kim, 2004). Desde un prisma ambiental, esta modalidad turística contribuye a la protección de los hábitats locales, al desarrollo de prácticas agrícolas sostenibles y a la conservación de los recursos naturales (Barbieri, 2013).

República Dominicana es un destino consolidado en turismo de sol y playa «todo incluido» y uno de los principales destinos del Caribe (OMT, 2019), si bien los ingresos del turismo no han aliviado la pobreza y claramente no han logrado reducir la desigualdad en la distribución de la riqueza (Oviedo-García et al., 2019). Actualmente, junto al turismo, la industria agrícola es una de las más importantes de la República Dominicana, sobre todo en producción de cacao, azúcar o plátanos (Payano-Almanzar y Rodriguez, 2018; Gómez-Luciano, De Koning, Vriesekoop y Urbano, 2019).

\subsection{Revisión de la literatura}

Kasarda y Janowitz (1974) consideraron a la comunidad como una estructura social que comprende dimensiones ecológicas, institucionales y normativas. La comunidad puede ser comprendida como una red multifacética y complicada de lazos sociales, conformados por la asociación de la sociedad de masas. En los estudios de turismo, el apego a la comunidad se considera un elemento significativo, que crea impactos en el apoyo al desarrollo turístico (Adongo, Choe y Han, 2017). Por eso, los académicos del turismo lo han utilizado repetidamente en modelos de apoyo al turismo para examinar sus efectos sobre las percepciones de las comunidades sobre los impactos del turismo y actitudes con respecto a la industria del turismo (Adongo et al., 2017). El apego a la comunidad se explica como el fuerte sentimiento positivo, arraigo y sentido de pertenencia de la gente hacia la comunidad (Matarrita-Cascante, Stedman y Luloff, 2010). Una definición conceptual de apego comunitario puede referirse como el alcance de la integración social y la participación en la comunidad y el sentimiento que las personas tienen hacia su comunidad (McCool y Martin, 1994).

Lee (2013) investigó sobre el apoyo al desarrollo del turismo sostenible a partir del apego a la comunidad. Utilizó los beneficios y costos, el apego a la comunidad, la participación de la comunidad y el apoyo para el desarrollo del turismo sostenible. Los resultados del estudio revelaron que la participación y el apego de la comunidad afectaron el nivel del apoyo al desarrollo del turismo sostenible. El apego de la comunidad se correlacionó directa y positivamente con los beneficios percibidos y tenía una correlación indirecta y positiva con el apoyo de los residentes para el desarrollo del turismo sostenible. Sin embargo, la relación entre los costos percibidos del turismo y el apego comunitario fue insignificante. Además, los resultados mostraron que los beneficios percibidos del turismo tuvieron un impacto mediador entre el apego de la comunidad y el apoyo al desarrollo del turismo sostenible. Los resultados del estudio mostraron que la participación de la comunidad influyó en los beneficios percibidos del turismo, e indirectamente y significativamente afectó al apoyo hacia el desarrollo del turismo sostenible. También se descubrió que la participación de la comunidad afecta los costos percibidos del turismo y afecta de manera indirecta e insignificante al propio apoyo hacia el turismo sostenible.

Jurowski y Uysal (2002) consiguieron que la preocupación de la comunidad, el vínculo de la comunidad, las actitudes ecocéntricas, el uso de los recursos turísticos y el estado de la economía local influyeron en las percepciones de los residentes locales sobre los impactos y su apoyo final al turismo. Chen y Chen (2010) 
indicaron que cuando los residentes participan más en sus actividades comunitarias, parece que tienen un mayor apego a su comunidad. También, cuando se aumenta ese apego al aumentar las inversiones en la comunidad, las percepciones de los residentes locales sobre el desarrollo del turismo tienden a ser positivas (Chen y Chen, 2010).

Nicholas et al. (2009) también encontraron una correlación sustancial entre el apego a la comunidad y la percepción de los encuestados sobre el desarrollo del turismo sostenible. Estudios más recientes respaldaron esta relación como positiva; por ejemplo, los resultados de la investigación realizada por Adongo et al. (2017) demostraron que los encuestados con mayor apego tenían un sentimiento más positivo hacia los efectos del turismo. Del mismo modo, los resultados de otro estudio reciente realizado por Huong y Lee (2017) revelaron que los residentes locales con un fuerte apego a su comunidad tenían percepciones positivas sobre los impactos del turismo en términos de beneficios sociales, ambientales, económicos personales y locales, lo que resulta en un mayor apoyo al desarrollo turístico sostenible.

Para que el turismo sea sostenible, la participación de la comunidad en la conservación del medio ambiente siempre debe aumentarse, para que el sentido de pertenencia de la comunidad sea cada vez más alto en el mantenimiento de su territorio como un destino que pueda apoyar la vida de las personas de manera sostenible, incluida la actividad turística (Murni et al., 2019). Los resultados del estudio de Chinyele y Lwoga (2019) indican que existe una relación significativamente positiva entre la participación en la toma de decisiones y la actitud hacia la conservación. Dupke, Dormann y Heurich (2019) argumentaron que para mantener el equilibrio entre los objetivos duales de conservación y recreación, los administradores de áreas naturales o rurales necesitan el apoyo de las comunidades locales.

El análisis de estas variables ha servido para determinar cómo influye el turismo en el apoyo al desarrollo sostenible (Gu y Ryan, 2008; Lee, 2013; Eslami et al., 2019), aunque no han sido analizadas en el agroturismo. En este sentido, esta investigación pretendió analizar si el apego a la comunidad, las percepciones turísticas positivas y la participación en la conservación y en el turismo tienen relación con el apoyo al desarrollo del agroturismo sostenible en la República Dominicana. Se seleccionaron estas variables porque, debido a las características socioeconómicas y geográficas de la región estudiada, éstas podría ayudar a obtener datos relevantes para que los diferentes stakeholders puedan establecer políticas y estrategias para desarrollar esta actividad turística en la región noroeste de la República Dominicana.

\section{Metodología}

El universo de esta investigación está compuesto por los residentes locales de la región noroeste de la República Dominicana. Esta región está compuesta por cuatro provincias: Dajabón, Mao, Santiago Rodríguez y Montecristi. Se ha seleccionado esta zona porque es la principal región agroindustrial del país (Orgaz-Agüera y López-Guzmán, 2015). Según datos de la Oficina Nacional de Estadística (ONE, 2020), esta región cuenta con 377,307 habitantes, de los cuales se encuestaron a 560 (error muestral del 4.1\%). El instrumento consistió en un cuestionario estructurado, diseñado en escala de Likert de 5 puntos, donde 1 correspondía a "nada de acuerdo" y 5 a "muy de acuerdo». Para la elaboración del cuestionario, se determinaron los constructos del cuestionario, a partir de dos elementos: 1) revisión de la literatura en relación a los diferentes constructos estudiados; y 2) selección de los constructos que podrían generar conclusión según el contexto socioeconómico y geográfico de la zona objeto de estudio de esta investigación; luego se adaptaron, a partir de la literatura, los ítems correspondientes a cada constructo, se tradujeron los ítems al idioma español y, finalmente, se realizó un pre-test de 20 encuestas con la finalidad de detectar posibles problemas. El cuestionario se muestra en el cuadro 1. 
Cuadro 1

Secciones, constructos e ítems del cuestionario

\begin{tabular}{|c|c|c|c|c|}
\hline Sección & Constructo & $\begin{array}{c}\text { Códig } \\
0\end{array}$ & Ítems & Adaptado de \\
\hline \multirow{9}{*}{1} & \multirow{9}{*}{$\begin{array}{l}\text { Apego a la } \\
\text { comunidad }\end{array}$} & PA1 & Siento que esta región es parte de mí & Eusébio, Vieira y Lima (2018) \\
\hline & & PA2 & Esta región es muy especial para mí & Eusébio et al. (2018) \\
\hline & & PA3 & Me identifico fuertemente con esta región & Eusébio et al. (2018) \\
\hline & & PA4 & $\begin{array}{l}\text { Me siento muy conectado con esta región y con las personas } \\
\text { que viven aquí }\end{array}$ & Eusébio et al. (2018) \\
\hline & & PA5 & Esta región significa mucho para mí & Eusébio et al. (2018) \\
\hline & & PA6 & Extraño esta región cuando no estoy aquí & Eusébio et al. (2018) \\
\hline & & PA7 & Tengo sentimientos positivos hacia esta región & Rasoolimanesh et al. (2019) \\
\hline & & PA8 & Tengo un sentido de pertenencia por esta región & Rasoolimanesh et al. (2019) \\
\hline & & PA9 & $\begin{array}{l}\text { Tengo un apego emocional a esta región, que es muy } \\
\text { especial para mí }\end{array}$ & Rasoolimanesh et al. (2019) \\
\hline \multirow{3}{*}{2} & \multirow{3}{*}{$\begin{array}{l}\text { Participación } \\
\text { en } \\
\text { conservación } \\
\text { y agroturismo }\end{array}$} & PC1 & $\begin{array}{l}\text { La participación de los residentes en el agroturismo } \\
\text { mejoraría la conservación de la región }\end{array}$ & Latip et al. (2018) \\
\hline & & PC2 & $\begin{array}{c}\text { La participación de los residentes en el turismo mejoraría la } \\
\text { experiencia de los turistas }\end{array}$ & Latip et al. (2018) \\
\hline & & PC3 & $\begin{array}{l}\text { Los residentes pueden conservar mejor que los extranjeros } \\
\text { los recursos turísticos }\end{array}$ & Latip et al. (2018) \\
\hline \multirow{9}{*}{3} & \multirow{9}{*}{$\begin{array}{l}\text { Percepciones } \\
\text { agroturísticas } \\
\text { positivas }\end{array}$} & PP1 & El desarrollo del turismo atrae más inversión a mi región & Rasoolimanesh et al. (2019) \\
\hline & & PP2 & Nuestro nivel de vida aumenta con el desarrollo del turismo & Rasoolimanesh et al. (2019) \\
\hline & & PP3 & $\begin{array}{l}\text { El desarrollo del turismo mejora la infraestructura y servicios } \\
\text { públicos (carreteras, etc.) }\end{array}$ & Eusébio et al. (2018) \\
\hline & & PP4 & $\begin{array}{l}\text { El desarrollo del turismo ayuda a preservar los edificios } \\
\text { históricos }\end{array}$ & Rasoolimanesh et al. (2019) \\
\hline & & PP5 & El desarrollo del turismo mejora la apariencia de la región & Rasoolimanesh et al. (2019) \\
\hline & & PP6 & $\begin{array}{c}\text { El desarrollo del turismo mejora la identidad cultural de la } \\
\text { región }\end{array}$ & Rasoolimanesh et al. (2019) \\
\hline & & PP7 & $\begin{array}{l}\text { El desarrollo del turismo promueve el intercambio cultural } \\
\text { entre residentes y turistas }\end{array}$ & Rasoolimanesh et al. (2019) \\
\hline & & PP8 & $\begin{array}{l}\text { El desarrollo del turismo mejora las oportunidades de ocio y } \\
\text { recreación en la región }\end{array}$ & Eusébio et al. (2018) \\
\hline & & PP9 & $\begin{array}{l}\text { El turismo contribuye a mejorar la reputación de mi región } \\
\text { en el extranjero }\end{array}$ & Martín et al. (2018) \\
\hline \multirow{6}{*}{4} & \multirow{6}{*}{$\begin{array}{l}\text { Apoyo al } \\
\text { desarrollo del } \\
\text { agroturismo } \\
\text { sostenible }\end{array}$} & ST1 & Me gustaría ver más turistas en la región & Nunkoo y So (2015) \\
\hline & & $\mathrm{ST} 2$ & Me gustaría visitar lugares turísticos en mi región & Rasoolimanesh et al. (2017) \\
\hline & & ST3 & Creo que el turismo debe fomentarse más en mi región & Rasoolimanesh et al. (2017) \\
\hline & & ST4 & Apoyo el desarrollo del turismo & Nunkoo y So (2015) \\
\hline & & ST5 & $\begin{array}{l}\text { Me gustaría que el turismo se convirtiera en parte } \\
\text { importante para mi región }\end{array}$ & Nunkoo y So (2015) \\
\hline & & ST6 & $\begin{array}{c}\text { El Gobierno Local debe fomentar más el desarrollo del } \\
\text { turismo en la región }\end{array}$ & Rasoolimanesh et al. (2017) \\
\hline 5 & $\begin{array}{l}\text { Perfil } \\
\text { sociodemo- } \\
\text { gráfico }\end{array}$ & F1-F9 & $\begin{array}{c}\text { Género, lugar de residencia, edad, estudios, sueldo, } \\
\text { habitantes en casa, actividad laboral, actividad agrícola, y } \\
\text { actividad turística }\end{array}$ & Elaboración propia \\
\hline
\end{tabular}

Fuente: elaboración propia

El cuestionario fue aplicado durante los meses de septiembre a noviembre de 2019 (ambos inclusive) en las cuatro provincias. Para el estudio descriptivo de los ítems, los datos tabulados se analizaron mediante el programa IBM SPSS v.24. 
Para la construcción del modelo estructural se partió de una revisión de la literatura con el fin de justificar las relaciones existentes entre los distintos constructos que componen el modelo (Apego a la comunidad, participación en conservación y agroturismo, percepciones agrturísticas positivas y apoyo al desarrollo del agroturismo sostenible) (ver figura 1). Para la validación del modelo estructural, los datos tabulados se analizaron mediante el programa Smart-PLS v.3.2.8. La evaluación de las hipótesis a través de una ecuación estructural lineal implica que se podrían llevar a cabo relaciones entre los constructos para determinar el poder predictivo del modelo.

Figura 1

Modelo estructural propuesto

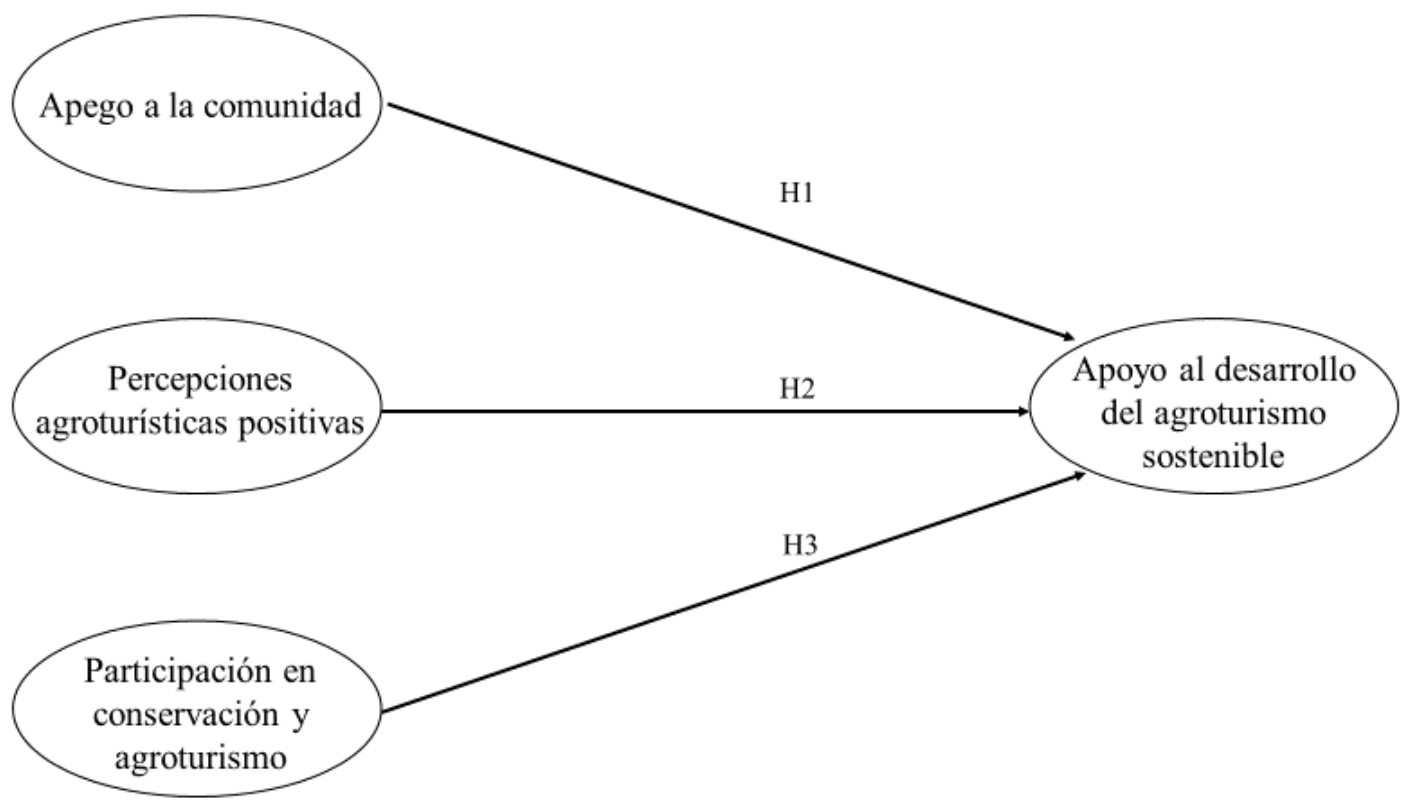

Fuente: elaboración propia

El análisis del modelo se fundamentó en una triple valoración, iniciada con el análisis del modelo global, seguido del análisis de fiabilidad y validez del modelo de medida y, finalmente, a través del análisis del modelo estructural. Para el análisis del ajuste del modelo global se ha acudido al cálculo de la Raíz Cuadrada Media Residual Estandarizada (SRMR), arrojando un valor de 0,054. En este sentido, valores de SRMR inferiores a 0,08 indican un grado de ajuste considerable (Hu y Bentler, 1998; Henseler, 2017).

Una vez analizado el modelo global, la fiabilidad y validez del modelo de medida es medida a través de las cargas factoriales (Compuestos Modo A), implicando valores superiores a 0,707 que la varianza compartida entre el constructo y sus indicadores es mayor que la varianza del error (Ali et al., 2018). Han sido incluidas cargas inferiores a 0,707, las cuales son aceptadas en etapas iniciales de investigaciones (Chin, 1998). Por otro lado, los Compuestos Modo B son medidos a través de los pesos. En este sentido, los pesos no significativos, pero con una carga externa superior a 0,5 han de ser mantenidos (Hair, Hult Ringle y Sarstedt, 2017). En el presente modelo, dos de los ítems relativos al compuesto Modo B fueron eliminados ya que no cumplían el requisito anterior. La existencia de multicolinealidad en los compuestos Modo B se ha comprobado a través del test de Factor de Inflación de la Varianza (VIF, en adelante) suponiendo valores de VIF superiores a 3,3 problemas de multicolinealidad (Roberts y Thatcher, 2009) (cuadro 2).

La fiabilidad del constructo es validado y medido a través de la fiabilidad compuesta de Dillon-Goldstein ( $\rho$ ) y la fiabilidad compuesta de Dijkstra-Henseler ( $\rho$ A) (Dijkstra y Henseler, 2015), siendo ambos mejores instrumentos que el alfa de Cronbach debido a que estas no tienen en cuenta el número de ítems que puede tener la escala 
(Hair, Sarstedt, Hopkins y Kuppelwieser, 2014). La fiabilidad compuesta acepta valores que van desde 0,6 (Diamantopoulos y Siguaw, 2000) a 0,8 (Koufteros, 1999). Todos los constructos presentes en el modelo presentaron valores superiores a 0,7 , incluso algunos superaron la barrera de 0,9 (cuadro 2).

La validez convergente y la validez discriminante es calculada para valorar la validez global de los compuestos. Para ello, se acude a la AVE para estimar la validez convergente, debiéndose obtener valores superiores a 0,5 para la existencia de validez convergente (Fornell y Larcker, 1981). Por otro lado, se acude a la ratio HeterotraitMonotrait (HT-MT, en adelante) para la validación de la validez discriminante, siendo este método el mejor indicado (Henseler, Ringle y Sarstedt, 2015). Valores inferiores a 0,85 (Kline, 2011) indicaría existencia de validez discriminante) (cuadro 3).

El modelo estructural fue examinado a través del coeficiente de determinación y la varianza explicada de las variables dependientes, poniendo esto de manifiesto la fuerza de las relaciones entre los constructos. Además, se llevó a cabo un bootstrapping para contrastar las hipótesis planteadas en el modelo. El cuadro 4 indica la cantidad de varianza explicada.

En relación con el contraste de hipótesis (tabla 5), se llevó a cabo un bootstrapping de 10000 submuestras (Streukens y Leroi-Werelds, 2016) para la obtención de los intervalos de confianza (vía no paramétrica), así como la significancia estadística de los coeficientes a través de la t de student (vía paramétrica) (Henseler, Ringle y Sinkovics, 2009).

El contraste de hipótesis realizado pone de manifiesto la influencia de cada una de las variables exógenas (Apego a la comunidad, Participación en conservación y agroturismo y percpciones agroturísticas positivas) sobre la variable endógena del modelo: apoyo al desarrollo del agroturismo sostenible

\section{Resultados}

\section{Cuadro 2}

Análisis de validez y fiabilidad de los Compuestos Modo A y Modo B.

\begin{tabular}{|c|c|c|c|c|c|c|}
\hline \multirow{2}{*}{ Constructo } & \multicolumn{3}{|c|}{ Fiabilidad individual } & \multicolumn{3}{|c|}{ Fiabilidad del compuesto } \\
\hline & Carga (p.lim) & Peso (p.lim) & VIF & $\rho c$ & $\rho A$ & AVE \\
\hline $\begin{array}{l}\text { Apego a la comunidad }(\mathrm{PA})-(\text { Modo } A) \\
\qquad A 1 \\
\text { PA2 } \\
\text { PA3 } \\
\text { PA4 } \\
\text { PA5 } \\
\text { PA6 } \\
\text { PA7 } \\
\text { PA8 } \\
\text { PA9 }\end{array}$ & $\begin{array}{l}0,740(0,000) \\
0,796(0,000) \\
0,855(0,000) \\
0,739(0,000) \\
0,749(0,000) \\
0,641(0,000) \\
0,730(0,000) \\
0,601(0,000) \\
0,659(0,000)\end{array}$ & $\mathrm{n} / \mathrm{a}$ & $\mathrm{n} / \mathrm{a}$ & 0,909 & 0,899 & 0,529 \\
\hline $\begin{array}{l}\text { Participación en conservación y } \\
\text { agroturismo (PC) - (Modo A) } \\
\qquad \begin{array}{c}P C 1 \\
P C 2 \\
P C 3\end{array}\end{array}$ & $\begin{array}{l}0,828(0,000) \\
0,861(0,000) \\
0,710(0,000)\end{array}$ & $\mathrm{n} / \mathrm{a}$ & $\mathrm{n} / \mathrm{a}$ & 0,867 & 0,816 & 0,521 \\
\hline $\begin{array}{l}\text { Apoyo al desarrollo del agroturismo } \\
\text { sostenible (ST) - (Modo A) } \\
\text { ST5 } \\
\text { ST6 } \\
\text { ST9 } \\
\text { ST10 }\end{array}$ & $\begin{array}{l}0,680(0,000) \\
0,705(0,000) \\
0,724(0,000) \\
0,768(0,000)\end{array}$ & $\mathrm{n} / \mathrm{a}$ & $\mathrm{n} / \mathrm{a}$ & 0,843 & 0,755 & 0,644 \\
\hline
\end{tabular}




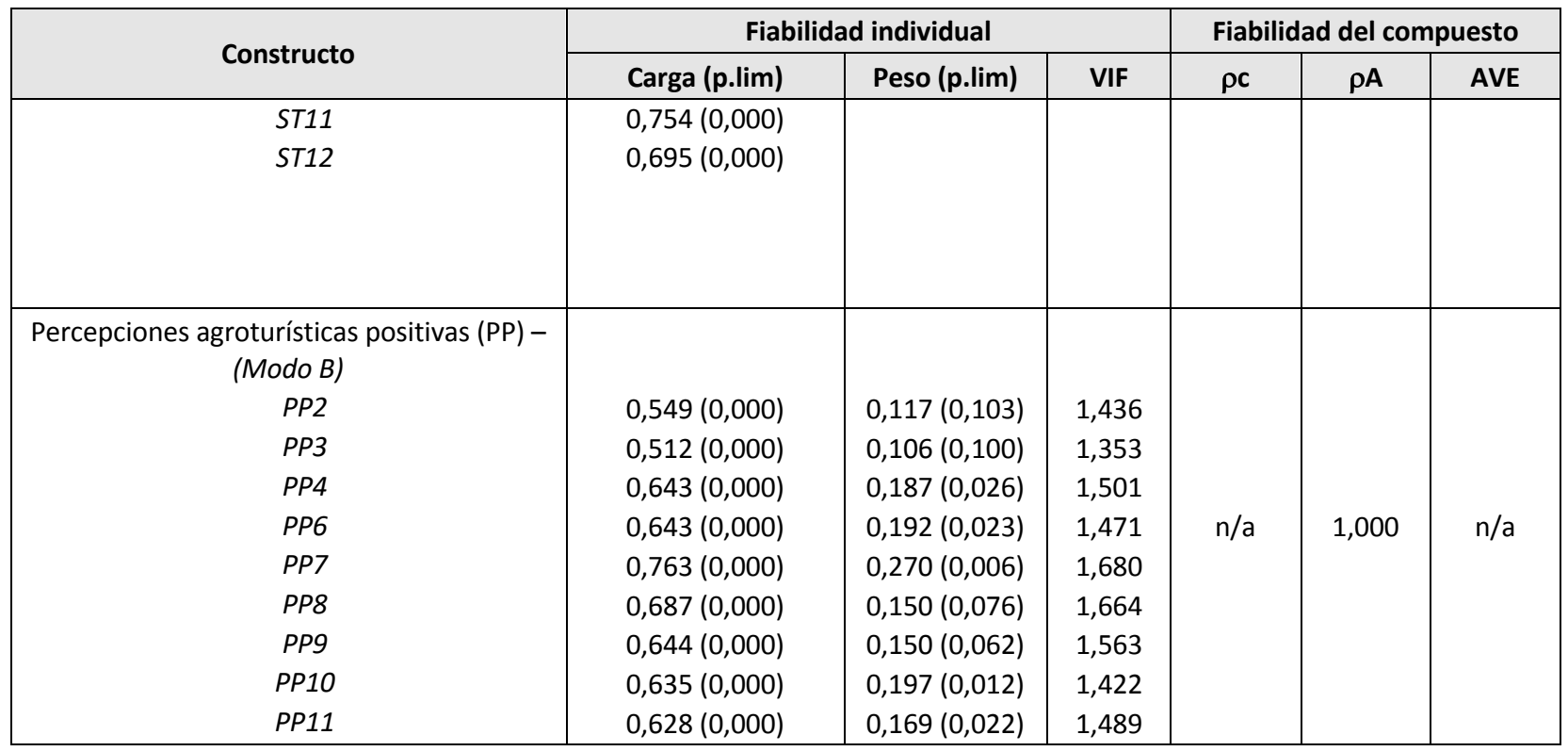

Fuente: elaboración propia. Notas: ${ }^{*} \mathrm{p}<0,05$ (t de student $(5000) ;$ una cola); $\mathrm{t}(0,05 ; 4999)=1,645$. VIF = Test de Factor de Inflación de la Varianza; rc = Fiabilidad compuesta de Dillon-Goldstein; Ra = Fiabilidad compuesta de Dijkstra-Henseler; $\mathrm{AVE}=$ Varianza Extraída Media. $\mathrm{n} / \mathrm{a}=$ no aplicable

Cuadro 3

Validez discriminante (Ratio HT-MT).

\begin{tabular}{|c|c|c|c|c|}
\hline & (1) & (2) & (3) & (4) \\
\hline (1) Apego a la comunidad (PA) & 0,727 & & & \\
\hline (2) Apoyo al desarrollo del agroturismo sostenible (ST) & 0,564 & 0,722 & & \\
\hline (3) Participación en conservación y agroturismo (PC) & 0,498 & 0,489 & 0,802 & \\
\hline (4) Percepciones agroturísticas positivas (PP) & 0,476 & 0,550 & 0,455 & \\
\hline
\end{tabular}

Fuente: elaboración propia

Cuadro 4

Efecto en las variables endógenas

\begin{tabular}{|c|c|c|c|c|}
\hline & $\mathbf{R}^{2}$ & $\begin{array}{l}\text { Efecto } \\
\text { directo }\end{array}$ & Correlación & $\begin{array}{l}\text { Varianza } \\
\text { Explicada }\end{array}$ \\
\hline Apoyo al desarrollo del agroturismo sostenible & 0,444 & & & \\
\hline H1: Apego a la comunidad & & 0,323 & 0,564 & $18,21 \%$ \\
\hline H2: Percepciones agroturísticas positivas & & 0,311 & 0,550 & $17,11 \%$ \\
\hline H3: Participación en conservación y agroturismo & & 0,186 & 0,489 & $9,09 \%$ \\
\hline
\end{tabular}

Fuente: elaboración propia

Así, la variable apego a la comunidad explica un $18,21 \%$ de la varianza de la variable apoyo al desarrollo de agroturismo sostenible. Por otro lado, las percepciones agroturísticas positivas y la participación en conservación y agroturismo explican un $17,11 \%$ y un $9,09 \%$ respectivamente de la variabilidad del apoyo al desarrollo de agroturismo sostenible. 
Cuadro 5

Hipótesis

\begin{tabular}{|l|c|c|c|c|c|}
\hline \multicolumn{1}{|c|}{ Hipótesis } & Efecto & Coeficiente & \multirow{2}{*}{$t$ (p.lim) } & \multicolumn{2}{|c|}{ IC (95\%) } \\
\cline { 4 - 6 } & sugerido & Path $(\beta)$ & $2,5 \%$ & $97,5 \%$ \\
\hline $\begin{array}{l}\text { H1: Apego a la comunidad } \rightarrow \text { Apoyo al desarrollo del } \\
\text { agroturismo sostenible }\end{array}$ & $(+/-)$ & $0,323 * * *$ & $\begin{array}{c}7,060 \\
(0,000)\end{array}$ & 0,232 & 0,412 \\
\hline $\begin{array}{l}\text { H2: Percepciones agroturísticas positivas } \rightarrow \text { Apoyo al } \\
\text { desarrollo del agroturismo sostenible }\end{array}$ & $(+/-)$ & $0,311^{* * *}$ & $\begin{array}{c}7,490 \\
(0,000)\end{array}$ & 0,246 & 0,408 \\
\hline $\begin{array}{l}\text { H3: Participación en conservación y agroturismo } \rightarrow \\
\text { Apoyo al desarrollo del agroturismo sostenible }\end{array}$ & $(+/-)$ & $0,186^{* * *}$ & $\begin{array}{c}4,463 \\
(0,000)\end{array}$ & 0,101 & 0,265 \\
\hline
\end{tabular}

Fuente: elaboración propia

Así, la primera de las hipótesis queda soportada, existiendo una influencia del apego a la comunidad sobre el apoyo al desarrollo del agroturismo sostenible $\left(\beta_{\mathrm{H} 1}=0,323 ; p=0,000\right)$. La segunda y la tercera de las hipótesis también confirman la influencia de las percepciones agroturísticas positivas $\left(H_{2}\right)$ y la participación en conservación y agroturismo $\left(\mathrm{H}_{3}\right)$ sobre el apoyo al desarrollo del agroturismo sostenible respectivamente $\left(\beta_{\mathrm{H}_{2}}=0,311 ; p=0,000\right)\left(\beta_{\mathrm{H} 3}=0,186 ; p=0,000\right)$ (cuadro 5 y figura 2$)$.

Figura 2

Modelo propuesto

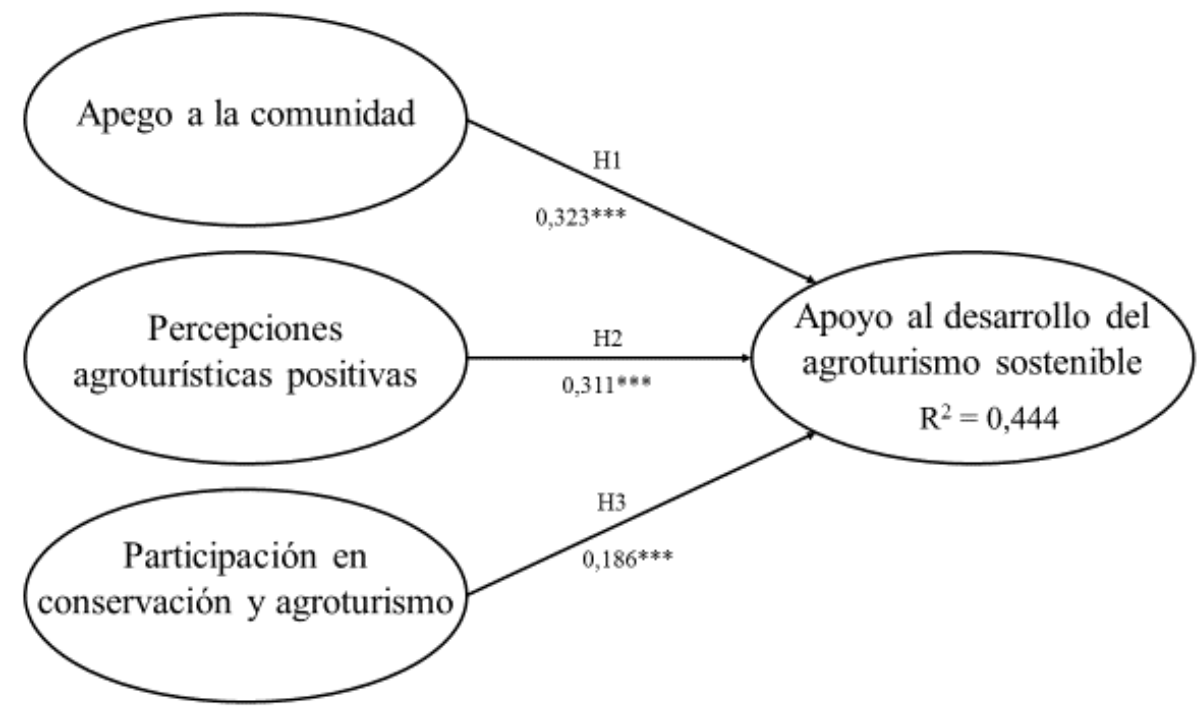

Fuente: elaboración propia

\section{Implicaciones y conclusiones}

Los resultados han confirmado que tanto el apego a la comunidad, las percepciones agroturísticas positivas como la participación en conservación y agroturismo influyen en el apoyo al desarrollo del agroturismo sostenible en la región noroeste de la República Dominicana. Este estudio muestra que el apego a la comunidad, las percepciones agroturísticas positivas, y la participación en conservación y agroturismo representan el 44,41 \% de la variabilidad del apoyo al desarrollo de la actividad agroturística sostenible. Sin embargo, en futuros estudios se deben abordar otras variables para conocer de qué forma explican el porcentaje restante de la variable apoyo al desarrollo del agroturismo sostenible.

La importancia teórica y práctica de este estudio se deriva del hecho de que conocer el apoyo con el que cuenta el desarrollo de la actividad agroturística puede fomentar también el desarrollo de la región noroeste de la República Dominicana; de esta manera, los resultados de este estudio son útiles para el Gobierno (Ministerio de 
Turismo y Ministerio de Agricultura, sobre todo). Estas instituciones deben apostar por implementar políticas y estrategias para impulsar el desarrollo del agroturismo sostenible en la región noroeste. Estas políticas y estrategias pueden contribuir al desarrollo de la región, que siendo una de las principales zonas agrícolas del país, poseen un impacto muy negativo en el desarrollo de la actividad turística. Así, compaginar el turismo con la agricultura puede ser muy beneficioso para la región, ya que se podría incentivar la economía más allá de estos sectores. Esto último es muy importante, debido a que esta región, además, poseen niveles de pobreza significativos.

Como todo trabajo de investigación, este estudio contempla algunas limitaciones. En este sentido, cabe destacar que la investigación está planteada desde la perspectiva del residente, dificultando la obtención de datos de otros grupos de stakeholders relevantes. Como futura línea de investigación, sería interesante introducir nuevas variables externas que ayuden a conocer la varianza explicada del apoyo al desarrollo del agroturismo sostenible.

\section{Referencias bibliográficas}

Ali, F., Rasoolimanesh, S. M., Sarstedt, M., Ringle, C., y Ryu, K. (2018). An assessment of the use of partial least squares structural equation modeling (PLS-SEM) in hospitality research. International Journal of Contemporary Hospitality Management, 30(1), 514-538.

Adongo, R., Choe, J. Y., y Han, H. (2017). Tourism in Hoi An, Vietnam: Impacts, perceived benefits, community attachment and support for tourism development. International Journal of Tourism Sciences, 17, 86-106.

Barbieri, C. (2013). Assessing the sustainability of agritourism in the US: A comparison between agri-tourism and other farm entrepreneurial ventures. Journal of Sustainable Tourism, 21(2), 252-270.

Chen, C.-F., y Chen, P.-C. (2010). Resident attitudes toward heritage tourism development. Tourism Geographies, 12, 525-545.

Chin, W. W. (1998). The partial last squares approach to structural equation modeling. En G. A. Marcoulides (Ed.). Modern methods for business research (pp. 295-336). Mahwah, Lawrence Erlbaum Associates

Chinyele, B. J., y Lwoga, N. B. (2019). Participation in decision making regarding the conservation of heritage resources and conservation attitudes in Kilwa Kisiwani, Tanzania. Journal of Cultural Heritage Management and Sustainable Development, 9(2), 184-198.

Diamantopoulos, A., y Siguaw, J. A. (2000). Introducing LISREL. London, SAGE Publications.

Dijkstra, T. K., y Henseler, J. (2015). Consistent partial least squares path modeling. MIS Quarterly, 39(2), 297316.

Dupke, C., Dormann, C.F., y Heurich, M. (2019). Does Public Participation Shift German National Park Priorities Away from Nature Conservation?. Environmental Conservation, 46, 84-91.

Eusébio, C., Vieira, A. L., y Lima, S. (2018). Place attachment, host-tourist interactions, and residents' attitudes towards tourism development: The case of Boa Vista Island in Cape Verde. Journal of Sustainable Tourism, 26(6), 890-909.

Eslami, S., Khalifah, Z., Mardani, A., Streimikiene, D., y Han, H. (2019). Community attachment, tourism impacts, quality of life and residents' support for sustainable tourism development. Journal of Travel \& Tourism Marketing, 36(9), 1061-1079. 
Fornell, C., y Larcker, D. F. (1981). Evaluating structural equation models with unobservable variables and measurement error. Journal of Marketing Research, 18,39-50.

Gómez-Luciano, C. A., De Koning, W., Vriesekoop, F., y Urbano, B. (2019). A model of agricultural sustainable added value chain: The case of the Dominican Republic value chain. Revista de la Facultad de Ciencias Agrarias UNCuyo, 51(1), 111-124.

Gu, H., y Ryan, C. (2008). Place attachment, identity and community impacts of tourism-the case of a Beijing hutong. Tourism Management, 29(4), 637-647.

Hair Jr, J. F., Hult, G. T. M., Ringle, C., y Sarstedt, M. (2017). A primer on partial least squares structural equation modeling (PLS-SEM). 2nd edition. Sage Publications.

Hair, J. F., Sarstedt, M., Hopkins, L., y Kuppelwieser, V. G. (2014). Partial Least Squares structural equation modeling (PLS-SEM). An emerging tool in business research. European Business Review, 26(2), 106-121.

Henseler, J. (2017). Bridging design and behavioral research with variance-based structural equation modeling. Journal of Advertising, 46(1), 178-192.

Henseler, J., Ringle, C.M. y Sarstedt, M. (2015). A new criterion for assessing discriminant validity in variancebased structural equation modeling. Journal of the Academy of Marketing Science, 43 (1), 115-135

Henseler, J., Ringle, C., y Sinkovics, R. (2009). The use of partial least squares path modeling in international marketing. Advances in International Marketing, 20, 277-319.

Hu, L-T., y Bentler, P.M. (1998). Fit indices in covariance structure modelling: Sensitivity to under parameterized model misspecification. Psychological Methods, 3(4), 424-453.

Huong, P. M., y Lee, J.-H. (2017). Finding important factors affecting local residents' support for tourism development in Ba Be National Park, Vietnam. Forest Science and Technology, 13, 126-132.

Ivars, J. (2000). Turismo y espacios rurales: conceptos, filosofías y realidades. Investigaciones geográficas, 23, 59-88.

Jurowski, C., Uysal, M., y Williams, D. R. (1997). A theoretical analysis of host community resident reactions to tourism. Journal of Travel Research, 36, 3-11.

Kasarda, J. D., y Janowitz, M. (1974). Community attachment in mass society. American Sociological Review, 3(39), 328-339.

Kline, R. (2011). Principles and practice of structural equation modelling (3rd ed.). New York, USA, Guilford Press.

Koufteros, X. A. (1999). Testing a model of pull production: A paradigm for manufacturing research using structural equation modeling. Journal of Operations Management, 17(4), 467-488.

Latip, N. A., Rasoolimanesh, S. M., Jaafar, M., Marzuki, A., y Umar, M. U. (2018). Indigenous participation in conservation and tourism development: A case of native people of Sabah, Malaysia. International Journal of Tourism Research, 20(3), 400-409.

Lee, T. H. (2013). Influence analysis of community resident support for sustainable tourism development. Tourism Management, 34, 37-46. 
Little, M. E., y Blau, E. (2019). Social adaptation and climate mitigation through agrotourism: a case study of tourism in Mastatal, Costa Rica. Journal of Ecotourism, 1-16.

Martín, H. S., de los Salmones Sanchez, M. M. G., y Herrero, Á. (2018). Residents' attitudes and behavioural support for tourism in host communities. Journal of Travel \& Tourism Marketing, 35(2), 231-243.

Matarrita-Cascante, D., Stedman, R., y Luloff, A. (2010). Permanent and seasonal residents' community attachment in natural amenity-rich areas: Exploring the contribution of landscape-related factors. Environment and Behavior, 42, 197-220.

McCool, S. F., y Martin, S. R. (1994). Community attachment and attitudes toward tourism development. Journal of Travel Research, 32, 29-34.

McGehee, N. G., y Kim, K. (2004). Motivation for agri-tourism entrepreneurship. Journal of Travel Research, 43, 161-170.

Murni, N. G. N. S., Ruki, M., y Antara, D. M. S. (2019). Model of Community Participation in Environmental Conservation to Support Sustainable Tourism. In International Conference On Applied Science and Technology 2019-Social Sciences Track (iCASTSS 2019). Atlantis Press.

Nicholas, L. N., Thapa, B., y Ko, Y. J. (2009). Residents' perspective of a world heritage site: The pitons management area, St. Lucia. Annals of Tourism Research, 36, 390-412.

Nunkoo, R., y So, K. K. F. (2015). Residents' support for tourism: Testing alternative structural models. Journal of Travel Research, 55(7), 847-861.

OMT (2019). Panorama del turismo internacional. Madrid, Organización Mundial del Turismo.

ONE (2020). Población de República Dominicana. Disponible en: https://www.one.gob.do/demograficas/proyecciones-de-poblacion

Orgaz-Agüera, F., y López-Guzmán, T. (2015). Análisis del perfil, motivaciones, y valoraciones de los turistas gastronómicos. El caso de la República Dominicana. ARA: Revista de Investigación en Turismo, 5(1), 43-52.

Oviedo-García, M. Á., González-Rodríguez, M. R., y Vega-Vázquez, M. (2019). Does sun-and-sea all-inclusive tourism contribute to poverty alleviation and/or income inequality reduction? The case of the Dominican Republic. Journal of Travel Research, 58(6), 995-1013.

Paül Carril, V., y Araújo Vila, N. (2012). Agroturismo en entornos periurbanos: enseñanzas de la iniciativa holeriturismo en el Parc Agrari del Baix Llobregat (Cataluña). Cuadernos de turismo, 29, 183-208.

Payano-Almanzar, R., y Rodriguez, J. (2018). Meteorological, Agricultural and Hydrological Drought in the Dominican Republic: A Review. Current World Environment, 13(1), 124-143.

Rasoolimanesh, S. M., Ringle, C. M., Jaafar, M., y Ramayah, T. (2017). Urban vs. rural destinations: Residents' perceptions, community participation and support for tourism development. Tourism Management, 60, 147-158.

Rasoolimanesh, S. M., Taheri, B., Gannon, M., Vafaei-Zadeh, A., y Hanifah, H. (2019). Does living in the vicinity of heritage tourism sites influence residents' perceptions and attitudes? Journal of Sustainable Tourism, 27(9), 1295-1317.

Roberts, N., y Thatcher, J. (2009). Conceptualizing and testing formative constructs: Tutorial and annotated example. ACM SIGMIS Database, 40, 3-39. 
Streukens, S., y Leroi-Werelds, S. (2016). Bootstrapping and PLS-SEM: A step-by-step guide to get more out of your bootstrap results. European Management Journal, 34, 618-632.

Zhen, L., Routray, J. K., Zoebisch, M. A., Chen, G., Xie, G., y Cheng, S. (2005). Three dimensions of sustainability of farming practices in the North China Plain: A case study from Ningjin County of Shandong Province, PR China. Agriculture, Ecosystems and Environment, 105(3), 507-522.

Zumbado-Morales, F. (2010). Agrotourism and agro-ecotourism in Costa Rica. e-Review of Tourism Research (eRTR), 8(6), 196-210.

\author{
Esta obra está bajo una Licencia Creative Commons \\ Attribución-NoCommercial 4.0 International \\ (cc) BY-Ne
}

